\title{
Erratum: Near-horizon geodesics of high spin black holes [Phys. Rev. D 101, 084042 (2020)]
}

\author{
Geoffrey Compère and Adrien Druart॰
}

(Received 4 June 2020; published 13 July 2020)

DOI: $10.1103 /$ PhysRevD.102.029901

(1) First, the captions of Figs. 1 and 2 should be inverted.

(2) Second, Eq. (2.24) should read as

$$
\Theta(z)= \begin{cases}\epsilon_{0}\left(\frac{Q}{\epsilon_{0}}+z\right)(1-z), & \epsilon_{0} \neq 0 \\ Q(1-z), & \epsilon_{0}=0,\end{cases}
$$

i.e., one should replace the $Q$ of the published version with $-Q$ in the first line of the right-hand side. This affects the discussion in items (a) and (b) of the subsequent paragraph as well as the content of Table 2 and Fig. 2 but nothing else. The corrected version of these items is provided below with replacements highlighted.

(a) $\epsilon_{0}>0$ leads to a polar motion $0 \leq z \leq 1$ for $Q>0$. For $Q=0$ and $z=0$, the motion is equatorial. For $Q=0$ and $z \neq 0, z=0$ is an asymptotic attractor of the motion which only takes place in one of the hemispheres. It is therefore a special case of equator-attractive motion where the turning point is at the pole $z=1$. For $Q<0$, the motion is either vortical $\left(0<-\frac{Q}{\epsilon_{0}} \leq z \leq 1\right)$ for $-\epsilon_{0}<Q<0$ or axial with $z=1$ for $Q \leq-\epsilon_{0}<0$.

(b) $\epsilon_{0}<0$ leads to a polar motion $0 \leq z \leq 1$ for $Q \geq-\epsilon_{0}>0$ and to a pendular one $\left(0 \leq z \leq-\frac{Q}{\epsilon_{0}}<1\right)$ for $0<Q<-\epsilon_{0}$. For $Q=0$, the motion is either equatorial or axial for the potential to be positive. For $Q<0$, the motion also has to take place along the axis $z=1$.

TABLE I. Polar taxonomy of Kerr geodesics with $\ell=0$.

\begin{tabular}{lccc}
\hline \hline Energy & Carter constant & Polar range & Denomination \\
\hline$\epsilon_{0}<0(|\hat{E}|<\mu)$ & $Q<0$ & $z=1$ & $\operatorname{Axial}^{0}(\hat{E}, Q)$ \\
& $Q=0$ & $z=0,1$ & $\operatorname{Equatorial}^{0}(\hat{E})$ \\
& $0<Q<-\epsilon_{0}$ & $\operatorname{Axial}^{0}(\hat{E})$ \\
& $0<-\epsilon_{0} \leq Q$ & $0 \leq z \leq-\frac{Q}{\epsilon_{0}}<1$ & $\operatorname{Pendular}^{0}(\hat{E}, Q)$ \\
& $-(a \hat{E})^{2} \leq Q<0$ & $0 \leq z \leq 1$ & $\operatorname{Polar}^{0}(\hat{E}, Q)$ \\
$\epsilon_{0}=0(|\hat{E}|=\mu)$ & $Q=0$ & $z=1$ & $\operatorname{Axial}_{\circ}^{0}(Q)$ \\
& $Q>0$ & $z=\operatorname{constant}$ & $\operatorname{Azimuthal}^{0}$ \\
& $Q \leq-\epsilon_{0}<0$ & $0 \leq z \leq 1$ & $\operatorname{Polar}_{0}^{0}(Q)$ \\
$\epsilon_{0}>0(|\hat{E}|>\mu)$ & $-\epsilon_{0}<Q<0$ & $z=1$ & $\operatorname{Axial}^{0}(\hat{E}, Q)$ \\
& $Q=0$ & $0<-\frac{Q}{\epsilon_{0}} \leq z \leq 1$ & $\operatorname{Vortical}^{0}(\hat{E}, Q)$ \\
& & $z \leq 1(\operatorname{sign}(\cos \theta)$ fixed $)$ & Equator-attractive $^{0}(\hat{E})$ \\
& $Q>0$ & $z=0$ & Equatorial $^{0}(\hat{E})$ \\
$\epsilon_{0} \in \mathbb{R}$ & $Q \geq-(a \hat{E})^{2}$ & $0 \leq z \leq 1$ & $\operatorname{Polar}^{0}(\hat{E}, Q)$ \\
\hline \hline
\end{tabular}




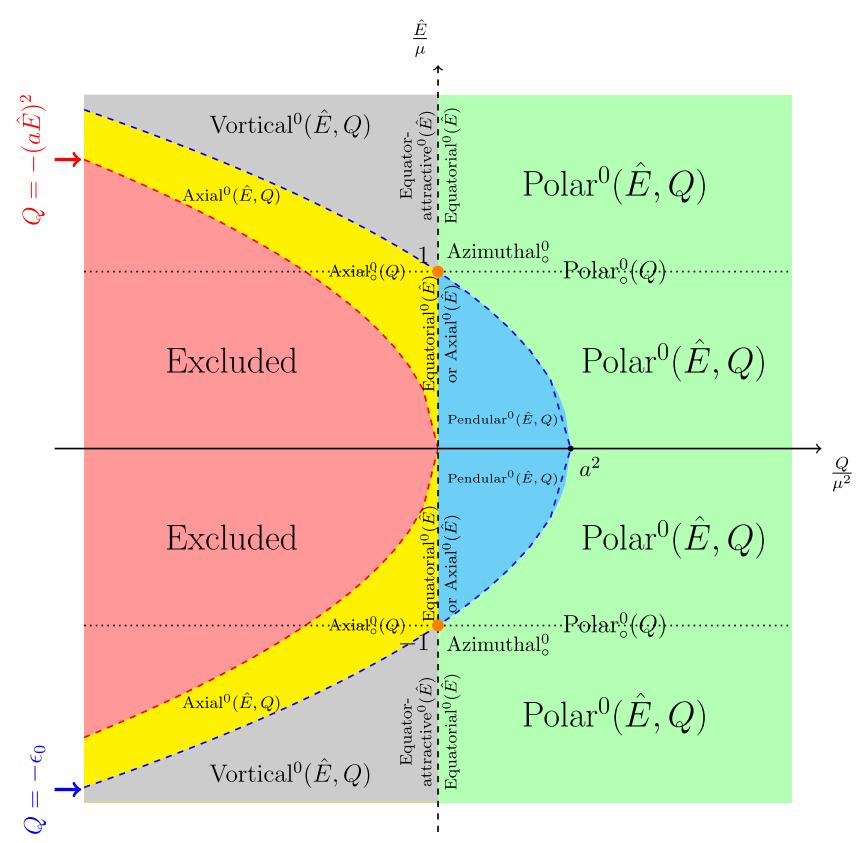

FIG. 1. Polar taxonomy of $\ell=0$ Kerr geodesics.

Let us finally notice that, for any value of $\varepsilon_{0}$ and $Q \geq-(a \hat{E})^{2}$, an axial motion is always possible.

The corrected polar classification of $\ell=0$ geodesics is provided in Table I. The taxonomy is also pictorially depicted in Fig. 1.

We thank Edward Teo for pointing out this mistake.

(3) Finally, there is a typo in Equation (2.37), which should read

$$
\hat{T}_{\theta}=\frac{-2 z_{+}}{\sqrt{-\varepsilon_{0} z_{-}}}\left[2 m E^{\prime}\left(\frac{z_{+}}{z_{-}}\right)+\left( \pm_{\theta}\right) E^{\prime}\left(\Psi^{+}(\cos \theta), \frac{z_{+}}{z_{-}}\right)-s_{\theta}^{i} E^{\prime}\left(\Psi^{+}\left(\cos \theta_{i}\right), \frac{z_{+}}{z_{-}}\right)\right] .
$$

